\title{
Excitation of solar-like oscillations across the HR diagram
}

\author{
R. Samadi ${ }^{1,2}$, D. Georgobiani ${ }^{3}$, R. Trampedach ${ }^{4}$, M. J. Goupil ${ }^{2}$, R. F. Stein ${ }^{5}$, and Å. Nordlund ${ }^{6}$ \\ 1 Observatório Astronómico UC, Coimbra, Portugal \\ e-mail: Reza.Samadi@obspm.fr \\ 2 Observatoire de Paris, LESIA, CNRS UMR 8109, 92195 Meudon, France \\ 3 Center for Turbulence Research, Stanford University NASA Ames Research Center, Moffett Field, USA \\ 4 Research School of Astronomy and Astrophysics, Mt. Stromlo Observatory, Cotter Road, Weston ACT 2611, Australia \\ 5 Department of Physics and Astronomy, Michigan State University, Lansing, USA \\ ${ }^{6}$ Niels Bohr Institute for Astronomy Physics and Geophysics, Copenhagen, Denmark
}

Received 6 September 2006 / Accepted 14 November 2006

\section{ABSTRACT}

\begin{abstract}
Aims. We extend semi-analytical computations of excitation rates for solar oscillation modes to those of other solar-like oscillating stars to compare them with recent observations

Methods. Numerical 3D simulations of surface convective zones of several solar-type oscillating stars are used to characterize the turbulent spectra as well as to constrain the convective velocities and turbulent entropy fluctuations in the uppermost part of the convective zone of such stars. These constraints, coupled with a theoretical model for stochastic excitation, provide the rate $\mathcal{P}$ at which energy is injected into the p-modes by turbulent convection. These energy rates are compared with those derived directly from the $3 \mathrm{D}$ simulations.

Results. The excitation rates obtained from the 3D simulations are systematically lower than those computed from the semi-analytical excitation model. We find that $\mathcal{P}_{\max }$, the $\mathcal{P}$ maximum, scales as $(L / M)^{s}$ where $s$ is the slope of the power law and $L$ and $M$ are the mass and luminosity of the 1D stellar model built consistently with the associated 3D simulation. The slope is found to depend significantly on the adopted form of $\chi_{k}$, the eddy time-correlation; using a Lorentzian, $\chi_{k}^{\mathrm{L}}$, results in $s=2.6$, whereas a Gaussian, $\chi_{k}^{\mathrm{G}}$, gives $s=3.1$. Finally, values of $V_{\max }$, the maximum in the mode velocity, are estimated from the computed power laws for $\mathcal{P}_{\max }$ and we find that $V_{\max }$ increases as $(L / M)^{s v}$. Comparisons with the currently available ground-based observations show that the computations assuming a Lorentzian $\chi_{k}$ yield a slope, $s v$, closer to the observed one than the slope obtained when assuming a Gaussian. We show that the spatial resolution of the 3D simulations must be high enough to obtain accurate computed energy rates.
\end{abstract}

Key words. convection - turbulence - Sun: oscillations - Hertzsprung-Russell (HR) and C-M - stars: variables: general methods: numerical

\section{Introduction}

Stars with masses $M \lesssim 2 M_{\odot}$ have upper convective zones where stochastic excitation of p-modes by turbulent convection takes place as in the case of the Sun. As such, these stars are often referred to as solar-like oscillating stars. One of the major goals of the future space seismology mission CoRoT (Baglin \& The Corot Team 1998), is to measure the amplitudes and the line-widths of these stochastically driven modes. From the measurements of the mode line-widths and amplitudes, it is possible to infer the rates at which acoustic modes are excited (see e.g. Baudin et al. 2005). Such measurements will then provide valuable constraints on the theory of stellar oscillation excitation and damping. In turn, improved models of excitation and damping will provide valuable information about convection in the outer layers of solar-like stars.

The mechanism of stochastic excitation has been modeled by several authors (e.g. Goldreich \& Keeley 1977; Osaki 1990; Balmforth 1992; Goldreich et al. 1994; Samadi \& Goupil 2001, for a review see Stein et al. 2004). These models yield the energy rate, $\mathcal{P}$, at which p-modes are excited by turbulent convection but require an accurate knowledge of the time averaged and - above all - the dynamic properties of turbulent convection.
Eddy time-correlations. In the approach of Samadi \& Goupil (2001, hereafter Paper I), the dynamic properties of turbulent convection are represented by $\chi_{k}$, the frequency component of the auto-correlation product of the turbulent velocity field; $\chi_{k}$ can be related to the convective eddy time-correlations. Samadi et al. (2003b, hereafter Paper III) have shown that the Gaussian function usually used for modeling $\chi_{k}$ is inappropriate and is at the origin of the under-estimation of the computed maximum value of the solar $\mathrm{p}$-modes excitation rates when compared with the observations. On the other hand, the authors have shown that a Lorentzian profile provides the best fit to the frequency dependency of $\chi_{k}$ as inferred from a 3D simulation of the Sun. Indeed, values of $\mathcal{P}$ computed with the model of stochastic excitation of Paper I and using a Lorentzian for $\chi_{k}=\chi_{k}^{\mathrm{L}}$ is better at reproducing the solar seismic observations whereas a Gaussian function, $\chi_{k}^{\mathrm{G}}$, under-estimates the amplitudes of solar p-modes. Provided that such a non-Gaussian model for $\chi_{k}$ is assumed, the model of stochastic excitation is - for the Sun - rather satisfactory. An open question, which we address in the present paper, is whether such non-Gaussian behavior also stands for other solar-like oscillating stars and what consequences arise for the theoretical excitation rates, $\mathcal{P}$.

Stochastic excitation in stars more luminous than the Sun. In the last five years, solar-like oscillations have been detected 
in several stars (see for instance the review by Bedding \& Kjeldsen 2003). Theoretical calculations result in an overestimation of their amplitudes (see Kjeldsen \& Bedding 2001; Houdek $\&$ Gough 2002). For instance, using Gough's (1976, 1977) nonlocal and time dependent treatment of convection, Houdek et al. (1999) have calculated expected values of $V_{\max }$, the maximum oscillation amplitudes, for different solar-like oscillating stars. Their calculations, based on a simplified excitation model, imply that $V_{\max }$ of solar-type oscillations scale as $(L / M)^{1.5}$ where $L$ and $M$ are the luminosity and mass of the star (see Houdek \& Gough 2002, hereafter HG02). A similar scaling law was empirically found earlier by Kjeldsen \& Bedding (1995). As pointed out by HG02, all these scaling laws overestimate the observed amplitudes of solar-like oscillating stars hotter and more massive than the Sun (e.g. $\beta$ Hydri, $\eta$ Bootis, Procyon, $\xi$ Hydrae). As the mode amplitude results from a balance between excitation and damping, this overestimation of the mode amplitudes can be attributed either to an overestimation of the excitation rates or an underestimation of the damping rates. In turn, any overestimation of the excitation rates can be attributed either to the excitation model itself or to the underlying convection model.

All the related physical processes are complex and difficult to model. The present excitation model therefore uses a number of approximations such as the assumption of incompressibility, and the scale length separation between the modes and the turbulent eddies exciting the modes. It has been shown that the current excitation model is valid in the case of the Sun (Paper III), but its validity in a broader region of the HR-diagram has not been confirmed until now.

Testing the validity of the theoretical model of stochastic excitation with the help of 3D simulations of the outer layers of stellar models is the main goal of the present paper. For that purpose, we compare the p-mode excitation rates for stars with different temperatures and luminosities as obtained by direct calculations and by the semi-analytical method as outlined below.

Numerical 3D simulations enable one to compute directly the excitation rates of $\mathrm{p}$-modes for stars with various temperatures and luminosities. For instance this was already undertaken for the Sun by Stein \& Nordlund (2001) using the numerical approach introduced in Nordlund \& Stein (2001). Such calculations will next be called "direct calculations". They are timeconsuming and do not easily allow massive computations of the excitation rates for stars with different temperatures and luminosities. On the other hand, an excitation model offers the advantage of testing separately several properties entering the excitation mechanism which are not well understood or modeled. Furthermore, once it is validated, it can be used for a large set of 1D models of stars.

As it was done for the Sun in Samadi et al. (2003c, hereafter Paper II) and Paper III, 3D simulations can also provide quantities which can be implemented in a formulation for the excitation rate $\mathcal{P}$, thus avoiding the use of the mixing-length approach with the related free parameters, and assumptions about the turbulent spectra. Such calculations will next be called "semi-analytical calculations".

We stress however that in any case, we cannot avoid the use of 1D models for computing accurate eigen-frequencies for the whole observed frequency range. In the present paper, the $1 \mathrm{D}$ models are constructed to be as consistent as possible with their corresponding 3D simulations, as described in Sect. 3.

This paper is organized as follows: in Sect. 2 we present the methods considered here for computing $\mathcal{P}$, that is the so-called "direct" method based on Nordlund \& Stein's (2001) approach (Sect. 2.1) and the so-called "semi-analytical" method based on the approach from Paper I, with modifications as presented in Papers II and III and in the present paper (Sect. 2.2).

Comparisons between direct and semi-analytical calculations of the excitation rates are performed in seven representative cases of solar-like oscillating stars. The seven 3D simulations all have the same number of mesh points. Section 3 describes these simulations and their associated 1D stellar models.

The 3D simulations provide constraints on quantities related to the convective fluctuations, in particular the eddy timecorrelation function, $\chi_{k}$, which, as stressed above, plays an important role in the excitation of solar p-modes. The function $\chi_{k}$ is therefore inferred from each simulation and compared with simple analytical function (Sect. 4).

Computations of the excitation rates of their associated p-modes are next undertaken in Sect. 5 using both the direct approach and the semi-analytical approach. In the semianalytical method, we employ model parameters as derived from the 3D simulations in Sect. 4.

In Sect. 5.2 we derive the expected scaling laws for $\mathcal{P}_{\max }$, the maximum in $\mathcal{P}$, as a function of $L / M$ with both the direct and semi-analytical methods and compare the results. This allows us to investigate the implications of such power laws for the expected values of $V_{\max }$ and to compare our results with the seismic observations of solar-like oscillations in Sect. 5.3. We also compare with previous theoretical results (e.g. Kjeldsen \& Bedding 1995; Houdek \& Gough 2002).

We finally assess the validity of the present stochastic excitation model and discuss the importance of the choice of the model for $\chi_{k}$ in Sect. 6.

\section{Calculation of the p-mode excitation rates}

\subsection{The direct method}

The energy input per unit time into a given stellar acoustic mode is calculated numerically according to Eq. (74) of Nordlund \& Stein (2001) multiplied by $\mathcal{S}$, the area of the simulation box, to get the excitation rate (in $\operatorname{erg~s}^{-1}$ ):

$\mathcal{P}\left(\omega_{0}\right)=\frac{\omega_{0}^{2} \mathcal{S}}{8 \Delta v \mathcal{E}_{\omega_{0}}}\left|\int_{r} \mathrm{~d} r \Delta \hat{P}_{\mathrm{nad}}\left(r, \omega_{0}\right) \frac{\partial \xi_{\mathrm{r}}}{\partial r}\right|^{2}$

where $\Delta \hat{P}_{\text {nad }}(r, \omega)$ is the discrete Fourier component of the nonadiabatic pressure fluctuations, $\Delta P_{\text {nad }}(r, t)$, estimated at the mode eigenfrequency $\omega_{0}=2 \pi v_{0}, \xi_{\mathrm{r}}$ is the radial component of the mode displacement eigenfunction, $\Delta v=1 / T$ the frequency resolution corresponding to the total simulation time $T$ and $\mathcal{E}_{\omega_{0}}$ is the mode energy per unit surface area defined in Nordlund \& Stein (2001, their Eq. (63)) as:

$\mathcal{E}_{\omega_{0}}=\frac{1}{2} \omega_{0}^{2} \int_{r} \mathrm{~d} r \xi_{\mathrm{r}}^{2} \rho\left(\frac{r}{R}\right)^{2}$

Note that Eq. (1) corresponds to the direct calculation of $P d V$ work of the non-adiabatic gas and turbulent pressure (entropy and Reynolds stress) fluctuations on the modes. The energy in the denominator of Eq. (1) is essentially the mode mass. The additional factor which turns it into energy is the mode squared amplitude which is arbitrary and cancels the mode squared amplitude in the numerator. For a given driving (i.e. $P \mathrm{~d} V$ work), the variation of the mode energy is inversely proportional to the mode energy (see Sect. 3.2 of Nordlund \& Stein 2001). Hence, for a given driving, the larger the mode energy (i.e., the mode mass or mode inertia) the smaller the excitation rate. 
In Eq. (1) the non-adiabatic Lagrangian pressure fluctuation, $\Delta \hat{P}_{\text {nad }}(r, \omega)$, is calculated as the following: we first compute the non-adiabatic pressure fluctuations $\Delta P_{\text {nad }}(r, t)$ according to Eq. (A.3) in Appendix A. We then perform the temporal Fourier transform of $\Delta P_{\text {nad }}(r, t)$ at each depth $r$ to get $\Delta \hat{P}_{\text {nad }}(r, \omega)$.

The mode displacement eigenfunction $\xi_{\mathrm{r}}(r)$ and the mode eigenfrequency $\omega_{0}$ are calculated as explained in Sect. 3. Its vertical derivative, $\partial \xi_{\mathrm{r}} / \partial r$, is normalized by the mode energy per unit surface area, $\mathcal{E}_{\omega_{0}}$, and then multiplied by $\Delta \hat{P}_{\text {nad }}$. The result is integrated over the simulation depth, squared and divided by $8 \Delta v$. We next multiply the result by the area of the simulation box $(\mathcal{S})$ to obtain $\mathcal{P}$, the total excitation rates in $\operatorname{erg~s}^{-1}$ for the entire star. Indeed the nonadiabatic pressure fluctuations are uncorrelated on large scales, so that average $\Delta P_{\text {nad }}^{2}$ is inversely proportional to the area. Multiplication by the area of the stellar simulation gives the excitation rates for the entire star as long as the domain size is sufficiently large to include several granules.

\subsection{The semi-analytical method}

Calculations of excitation rates by the semi-analytical method are based on a model of stochastic excitation. The excitation model we consider is the same as presented in Paper I. In this model of excitation and in contrast to previous models (e.g. Goldreich \& Keeley 1977; Balmforth 1992; Goldreich et al. 1994), the driving by turbulent convection is ensured not only by the Reynolds stress tensor but also by the advection of the turbulent fluctuations of entropy by the turbulent movements (the so-called entropy source term).

As in Paper I, we consider only radial p-modes. We do not expect any significant differences for low $\ell$ degree modes. Indeed, in the region where the excitation takes place, the low $\ell$ degree modes have the same behavior as the radial modes. Only for very high $\ell$ degree modes $(\ell \gg 100)$ - which will not be seen in stars other than the Sun - can a significant effect be expected, as is quantitatively confirmed (work in progress).

The excitation rates are computed as in Paper II, except for the change detailed below. The rate at which a given mode with frequency $\omega_{0}=2 \pi v_{0}$ is excited is then calculated with the set of Eqs. (1)-(11) of Paper II. These equations are based on the excitation model of Paper I, but assume that injection of acoustic energy into the modes is isotropic. However, Eq. (10) of Paper II must be corrected for an analytical error (see Samadi et al. 2005). This yields the following correct expression for Eq. (10) of Paper II:

$$
\begin{aligned}
S_{R}\left(r, \omega_{0}\right)= & \int_{0}^{\infty} \frac{\mathrm{d} k}{k^{2}} \frac{E(k, r)}{u_{0}^{2}} \frac{E(k, r)}{u_{0}^{2}} \\
& \times \int_{-\infty}^{+\infty} \mathrm{d} \omega \chi_{k}\left(\omega_{0}+\omega, r\right) \chi_{k}(\omega, r)
\end{aligned}
$$

where $u_{0}=\sqrt{\Phi / 3} \bar{u}, \Phi$ is Gough's (1977) anisotropy factor, $\bar{u}$ is the rms value of $\boldsymbol{u}$, the turbulent velocity field, $k$ the wavenumber and $\chi_{k}(\omega)$ is the frequency component of the correlation product of $\boldsymbol{u}$.

The method then requires the knowledge of a number of input parameters which are of three different types:

1) Quantities which are related to the oscillation modes: the eigenfunctions $\left(\xi_{r}\right)$ and associated eigen-frequencies $\left(\omega_{0}\right)$.

2) Quantities which are related to the spatial and time averaged properties of the medium: the mean density $\left(\rho_{0}\right), \alpha_{s} \equiv$ $\left\langle(\partial p / \partial s)_{\rho}\right\rangle-$ where $s$ is the entropy, $p$ the gas pressure and $\langle\ldots\rangle$ denotes horizontal and time averages - the mean square of the vertical component of the convective velocity, $\left\langle w^{2}\right\rangle$, the mean square of the entropy fluctuations, $\left\langle\tilde{s}^{2}\right\rangle$, and the mean anisotropy, $\Phi$ (Eq. (2) of Paper II).

3) Quantities which contain information about spatial and temporal auto-correlations of the convective fluctuations: the spatial spectrum of the turbulent kinetic energy and entropy fluctuations, $E(k)$ and $E_{s}(k)$, respectively, as well as the temporal spectrum of the correlation product of the turbulent velocity field, $\chi_{k}$.

Eigen-frequencies and eigenfunctions (in 1) above) are computed with the adiabatic pulsation code ADIPLS (Christensen-Dalsgaard \& Berthomieu 1991) for each of the 1D models associated with the 3D simulations (see Sect. 3).

The spatial and time averaged quantities (in 2) and 3) above) are obtained from the 3D simulations in the manner of Paper II. For $E(k)$, however, we use the actual spectrum as calculated from the 3D simulations and not an analytical fit as was done in Paper II. However as in Paper II, we assume for $E_{s}(k)$ the $k$ dependency of $E(k)$ (we have checked this assumption for one simulation and found no significant change in $\mathcal{P}$ ).

For each simulation, we determine $\chi_{k}$ as in Paper III (cf. Sect. 4). Each $\chi_{k}$ is then compared with various analytical forms, among which some were investigated in Paper III. Finally we select the analytical forms which are the closest to the behavior of $\chi_{k}$ and use them, in Sect. 5, to compute $\mathcal{P}$.

\section{The convection simulations and their associated 1D models}

Numerical simulations of surface convection for seven different solar-like stars were performed by Trampedach et al. (1999). These hydrodynamical simulations are characterized by the effective temperature, $T_{\text {eff }}$ and acceleration of gravity, $g$, as listed in Table 1. The solar simulation with the same input physics and number of mesh points is included for comparison purposes. The surface gravity is an input parameter, while the effective temperature is adjusted by changing the entropy of the inflowing gas at the bottom boundary. The simulations have $50 \times 50 \times 82$ grid points. All of the models have solar-like chemical composition, with hydrogen abundance $X=0.703$ and metal abundance $Z=0.0145$. The simulation time-series all cover at least five periods of the primary p-modes (highest amplitude, one node at the bottom boundary), and as such should be sufficiently long.

The convection simulations are shallow (only a few percent of the stellar radius) and therefore contain only few modes. To obtain mode eigenfunctions, the simulated domains are augmented by $1 \mathrm{D}$ envelope models in the interior by means of the stellar envelope code by Christensen-Dalsgaard \& Frandsen (1983a). Convection in the envelope models is based on the mixing-length formalism (Böhm-Vitense 1958).

Trampedach et al. (2006a) fit 1D stellar envelopes to average stratifications of the seven convection simulations by matching temperature and density at a common pressure point near the bottom of the simulations. The fitting parameters are the mixinglength parameter, $\alpha$, and a form-factor, $\beta$, in the expression for turbulent pressure: $P_{\text {turb }}^{1 \mathrm{D}}=\beta \varrho u_{\mathrm{MLT}}^{2}$, where $u_{\mathrm{MLT}}$ is the convective velocities predicted by the mixing-length formulation. A consistent matching of the simulations and $1 \mathrm{D}$ envelopes is achieved by using the same equation of state (EOS) by Däppen et al. (1988, also referred to as the MHD EOS, with reference to Mihalas, Hummer, and Däppen) and opacity distribution functions (ODF) by Kurucz (1992a,b), and also by using $T-\tau$ relations derived from the simulations (Trampedach et al. 2006b). 
Table 1. Characteristics of the convection 3D simulations: $t_{\mathrm{sim}}$ is the duration of the relaxed simulations used in the present analysis, $H_{\mathrm{p}}$ is the pressure scale height at the surface, $L_{\mathrm{h}}$ the size of the box in the horizontal direction, $C_{\mathrm{s}}$ the sound speed and $t_{\mathrm{s}}$ the sound travel time across $H_{\mathrm{p}}$. All the simulations have a spatial grid of $50 \times 50 \times 82$.

\begin{tabular}{lccccccccc}
\hline \hline Star & $\begin{array}{c}t_{\text {sim }} \\
{[\mathrm{min}]}\end{array}$ & $\begin{array}{c}\text { size } \\
{\left[\mathrm{Mm}^{3}\right]}\end{array}$ & $\log g$ & $\begin{array}{c}T_{\text {eff }} \\
{[\mathrm{K}]}\end{array}$ & $\begin{array}{c}H_{\mathrm{p}} \\
{[\mathrm{km}]}\end{array}$ & $\begin{array}{c}L_{\mathrm{h}} / H_{p} \\
{\left[\mathrm{~km} \mathrm{~s}^{-1}\right]}\end{array}$ & $\begin{array}{c}C_{\mathrm{s}} \\
{[\mathrm{s}]}\end{array}$ & $t_{\text {sim }} / t_{\mathrm{s}}$ \\
\hline$\alpha$ Cen B & 59 & $4.0 \times 4.0 \times 2.2$ & 4.5568 & 5363 & 95. & 42.1 & 7.49 & 12.72 & 278.3 \\
Sun & 96 & $6.0 \times 6.0 \times 3.4$ & 4.4377 & 5802 & 134 & 44.8 & 7.78 & 17.30 & 332.9 \\
Star A & 80 & $11.6 \times 11.6 \times 6.4$ & 4.0946 & 4851 & 316 & 36.7 & 7.98 & 39.66 & 121.0 \\
$\alpha$ Cen A & 44 & $8.9 \times 8.8 \times 5.1$ & 4.2946 & 5768 & 189 & 47.1 & 7.81 & 24.17 & 109.2 \\
Star B & 110 & $20.7 \times 20.7 \times 11.3$ & 4.0350 & 6167 & 359 & 57.7 & 7.76 & 46.29 & 142.6 \\
Procyon & 119 & $20.7 \times 20.7 \times 10.9$ & 4.0350 & 6470 & 380 & 54.5 & 7.52 & 50.50 & 141.4 \\
$\eta$ Boo & 141 & $36.9 \times 36.9 \times 16.3$ & 3.7534 & 6023 & 709 & 52.0 & 7.40 & 96.13 & 88.0 \\
\hline
\end{tabular}

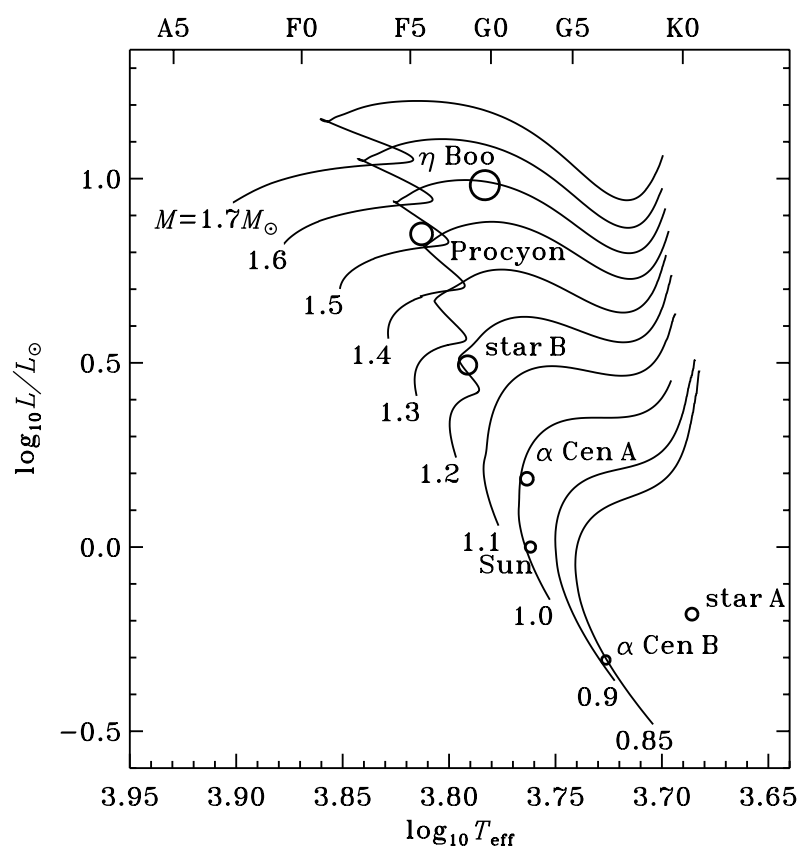

Fig. 1. Location of the convection simulations in the HR diagram. The symbol sizes vary proportionally to the stellar radii. Evolutionary tracks of stars, with masses as indicated, were calculated on the base of Christensen-Dalsgaard's stellar evolutionary code (Christensen-Dalsgaard 1982; Christensen-Dalsgaard \& Frandsen 1983a).

The average stratifications of the 3D simulations, augmented by the fitted $1 \mathrm{D}$ envelope models in the interior, were used as the basis for the eigenmode calculations using the adiabatic pulsation code by Christensen-Dalsgaard \& Berthomieu (1991). These combinations of averaged 3D simulations and matched $1 \mathrm{D}$ envelope models will, from hereon, be referred to as the 1D models.

The positions of the models in the HR diagram are presented in Fig. 1 and their global parameters are listed in Table 2. Five of the seven models correspond to actual stars, while Star A and Star B are merely sets of atmospheric parameters; their masses and luminosities are therefore assigned somewhat arbitrarily (the $L / M$-ratios, only depending on $T_{\text {eff }}$ and $g$, are of course not arbitrary).

\section{Inferred properties of $\chi_{k}$}

For each simulation, $\chi_{k}(\omega)$ is computed over the whole wavenumber $(k)$ range covered by the simulations and at different layers within the region where modes are excited. We present the results at the layer where the excitation is maximum,
Table 2. Fundamental parameters of the 1D-models associated with the 3D simulations of Table 1.

\begin{tabular}{lccccc}
\hline \hline Star & $T_{\text {eff }}$ & $M / M_{\odot}$ & $R / R_{\odot}$ & $L / L_{\odot}$ & $L M_{\odot} / M L_{\odot}$ \\
& {$[\mathrm{K}]$} & & & & \\
\hline$\alpha$ Cen B & 5363 & 0.90 & 0.827 & 0.51 & 0.56 \\
Sun & 5802 & 1.00 & 1.000 & 1.02 & 1.02 \\
Star A & 4852 & 0.60 & 1.150 & 0.66 & 1.10 \\
$\alpha$ Cen A & 5768 & 1.08 & 1.228 & 1.50 & 1.38 \\
Star B & 6167 & 1.24 & 1.769 & 4.07 & 3.28 \\
Procyon & 6470 & 1.75 & 2.102 & 6.96 & 3.98 \\
$\eta$ Boo & 6023 & 1.63 & 2.805 & 9.31 & 5.71 \\
\hline
\end{tabular}

i.e., where $u_{0}$ is maximum, and for two representative wavenumbers: $k=k_{\max }$ at which $E(k)$ peaks and $k=10 k_{\min }$, where $k_{\min }$ is the first non-zero wavenumber of the simulations. Indeed, the amount of acoustic energy going into a given mode is largest at this layer and at the wavenumber $k \simeq k_{\max }$, provided that the mode frequency satisfies: $\omega_{0} \lesssim\left(k_{\max } u_{0}\right)$. Above $\omega_{0} \sim k_{\max } u_{0}$, the efficiency of the excitation decreases rapidly. Therefore low and intermediate frequency modes (i.e., $\omega_{0} \lesssim k_{\max } u_{0}$ ) are predominantly excited at $k \simeq k_{\max }$. On the other hand, high frequency modes are predominantly excited by small-scale fluctuations, i.e. at large $k$. The exact choice of the representative large wavenumber is quite arbitrary; however it cannot be too large because of the limited number of mesh points $k \lesssim 25 k_{\min }$ and in any case, the excitation is negligible above $k \simeq 20 k_{\min }$. We thus chose the intermediate wavenumber $k=10 k_{\min }$. Figure 2 presents $\chi_{k}$ as obtained from the 3D simulations of Procyon, $\alpha$ Cen B and the Sun, at the layer where $u_{0}$ is maximum and for the wavenumber $k_{\max }$. Although defined as a function of $\omega$, for convenience, $\chi_{k}$ is plotted as a function of $v=\omega / 2 \pi$ throughout this paper. Figure 3 displays $\chi_{k}$ for $k=10 k_{\min }$. Results for the other simulations are not shown, as the results for Procyon, $\alpha$ Cen B and the Sun correspond to three representative cases.

In practice, it is not easy to implement directly in the excitation model the $v$-variation of $\chi_{k}$ inferred from the 3D simulations. An alternative and convenient way to compute $\mathcal{P}$ is to use simple analytical functions for $\chi_{k}$ which are chosen so as to best represent the $3 \mathrm{D}$ results. We then compare $\chi_{k}$ computed with the 3D simulations with the following simple analytical forms: the Gaussian form

$\chi_{k}^{\mathrm{G}}(\omega)=\frac{1}{\omega_{k} \sqrt{\pi}} \mathrm{e}^{-\left(\omega / \omega_{k}\right)^{2}}$,

the Lorentzian form

$\chi_{k}^{\mathrm{L}}(\omega)=\frac{1}{\pi \omega_{k} / 2} \frac{1}{1+\left(2 \omega / \omega_{k}\right)^{2}}$, 

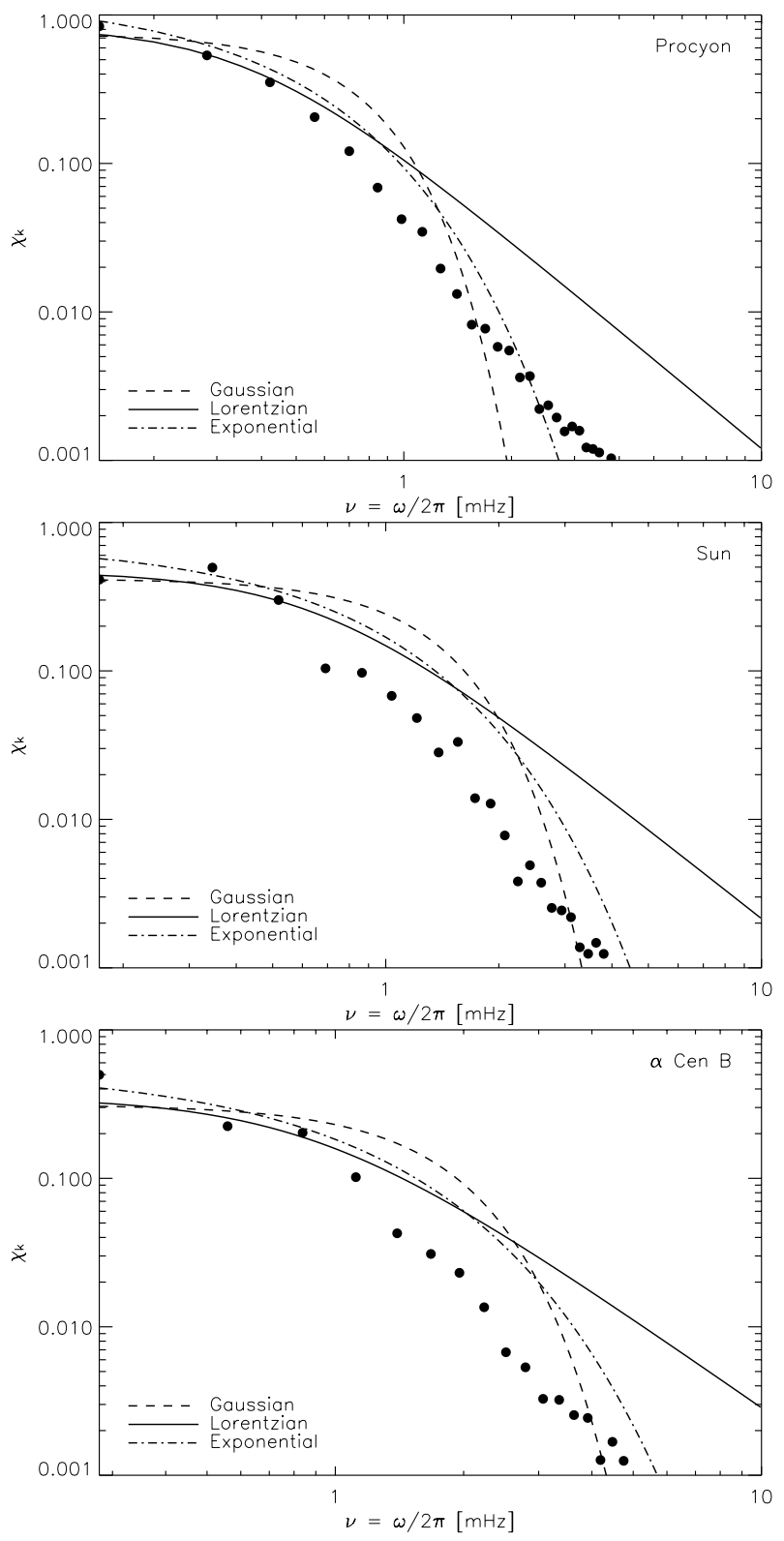

Fig. 2. The filled dots represent $\chi_{k}$ obtained from the 3D simulations for the wavenumber $k$ at which $E(k)$ is maximum and at the layer where the excitation is maximum in the simulation. The results are presented for three simulations: Procyon (top), the Sun (middle) and $\alpha$ Cen B (bottom). The solid curves represent the Lorentzian form, Eq. (5), the dashed curves the Gaussian form Eq. (4), and the dot dashed curves the exponential form Eq. (6).

and the exponential form

$\chi_{k}^{\mathrm{E}}(\omega)=\frac{1}{\omega_{k}} \mathrm{e}^{-\left|2 \omega / \omega_{k}\right|}$.

In Eqs. (4)-(6), $\omega_{k}$ is the line-width of the analytical function and is related to the velocity $u_{k}$ of the eddy with wave number $k$ as:

$\omega_{k} \equiv 2 k u_{k}$

In Eq. (7), $u_{k}$ is calculated from the kinetic energy spectrum $E(k)$ as (Stein 1967)

$u_{k}^{2}=\int_{k}^{2 k} \mathrm{~d} k E(k)$
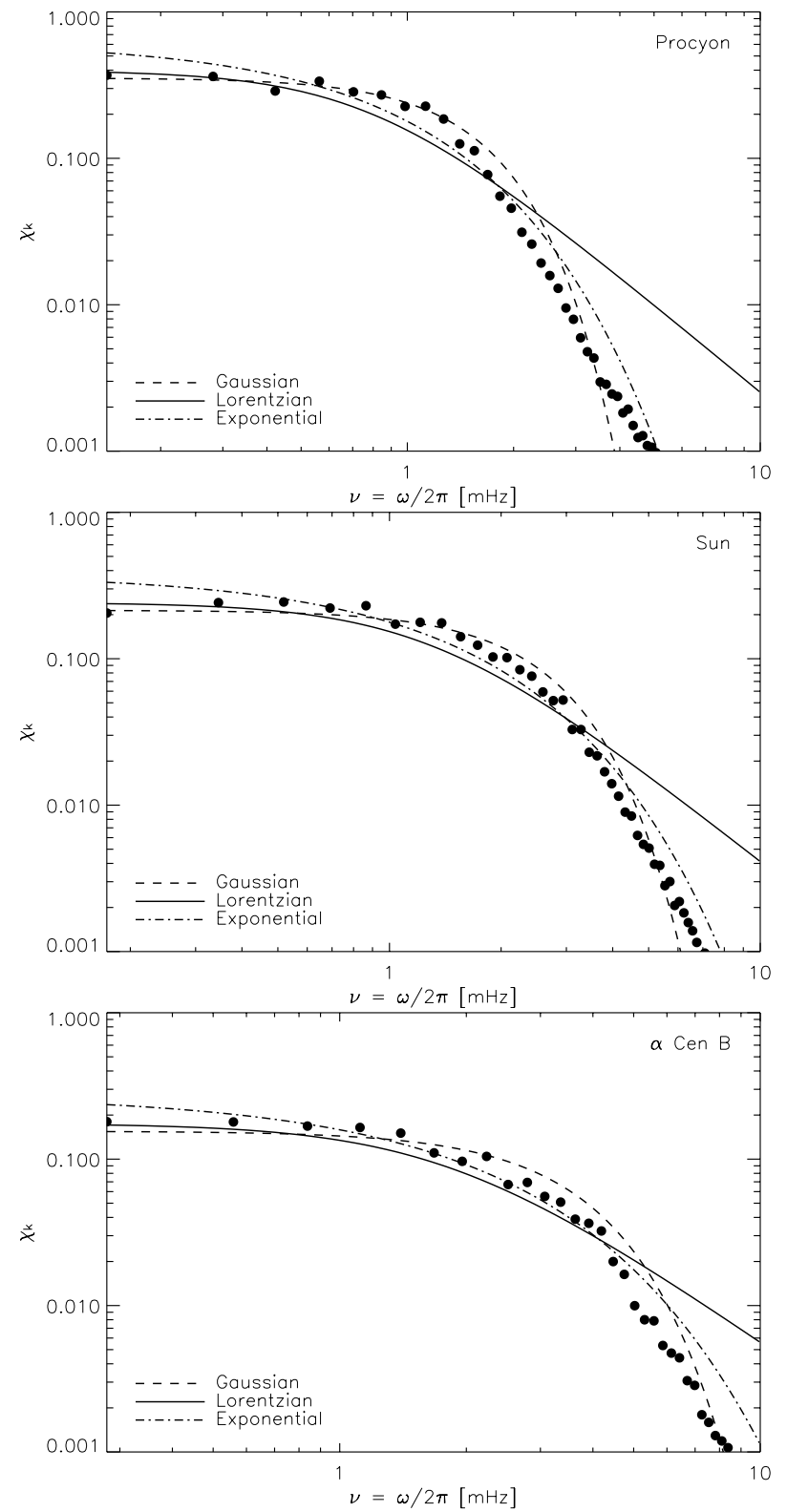

Fig. 3. Same as Fig. 2 for $k=10 k_{\min }$ where $k_{\min }$ is the first non-zero wavenumber of the simulation.

As shown in Figs. 2 and 3, the Lorentzian $\chi_{k}^{\mathrm{L}}$ does not reproduce the $v$-variation of $\chi_{k}$ satisfactorily. This is particularly true for the solar case. This contrast with the results of Paper III where it was found that $\chi_{k}^{\mathrm{L}}$ reproduces nicely - at the wavenumber where $E$ is maximum - the $v$-variation of $\chi_{k}$ inferred from the solar simulation investigated in Paper III. These differences in the results for the solar case can be explained by the low spatial resolution of the present solar simulation compared with that of Paper III. Indeed we have compared different solar simulations with different spatial resolution and found that the $v$-variation of $\chi_{k}$ converges to that of $\chi_{k}^{\mathrm{L}}$ as the spatial resolution increases (not shown here). This dependency of $\chi_{k}$ with spatial resolution of the simulation is likely to hold for the non-solar simulations as well. This result then suggests that $\chi_{k}$ is in fact best represented by the Lorentzian form, $\chi_{k}^{\mathrm{L}}$.

As a consequence, realistic excitation rates evaluated directly for a convection simulation should be based on simulations with higher spatial resolution. However the main goal of the present work is to test the excitation model, which can be done with the 
present set of simulations. Indeed, we only need to use as inputs for the excitation model the quantities related to the turbulent convection $\left(E(k), \chi_{k}, \ldots\right)$ as they are in the simulations, no matter how the real properties of $\chi_{k}$ are.

For the present set of simulations, we compare three analytical forms of $\chi_{k}$ : Lorentzian, Gaussian and exponential. For large $k, \chi_{k}$ is overall best modeled by a Gaussian (see Fig. 3 for $k=10 k_{\min }$ ). For small $k$ (see Fig. 2 for $k=k_{\max }$ ) both the exponential and the Gaussian are closer to $\chi_{k}$ than the Lorentzian.

For a given simulation, depending on the frequency, differences between $\chi_{k}(v)$ and the analytical forms are more or less pronounced.

The discrepancy between $\chi_{k}(v)$ inferred from the 3D simulations and the exponential or the Gaussian forms vary systematically with stellar parameters; decreasing as the convection gets more forceful, as measured by, e.g., the turbulent- to total-pressure ratio. Of the three simulations illustrated in Fig. 2, Procyon has the largest and $\alpha$ Cen B has the smallest $P_{\text {turb }} / P_{\text {tot }^{-}}$ ratio.

As a whole for the different simulations and scale lengths $k$, we conclude that the $v$-variation of $\chi_{k}$ in the present set of simulations lies between that of a Gaussian and an exponential. However, neither of them is completely satisfactory. Actually a recent detailed study by Georgobiani et al. (2006, in preparation) tends to show that $\chi_{k}$ cannot systematically be represented at all wavenumbers by a simple form such as a Gaussian, an exponential or a Lorentzian, but rather needs a more generalized power law. Hence, more sophisticated fits closer to the simulated $v$-variation of $\chi_{k}$ could have been considered, but for the sake of simplicity we chose to limit ourselves to the three forms presented here.

\section{5. p-mode excitation rates across the HR diagram}

\subsection{Excitation rate spectra $(\mathcal{P}(v))$}

For each simulation, the rates $\mathcal{P}$ at which the p-modes of the associated 1D models are excited are computed both directly from the 3D simulations and with the semi-analytical method (see Sect. 2). In this section, the semi-analytical calculations are based on two analytical forms of $\chi_{k}$ : a Gaussian and an exponential form as described in Sect. 4. The Lorentzian form as introduced in Sect. 4 is not investigated in the present section. Indeed our purpose here is to test the model of stochastic excitation by using constraints from the 3D simulations, and a Lorentzian behaviour is never obtained in the present $3 \mathrm{D}$ simulations.

The results of the calculations of $\mathcal{P}$ using both methods are presented in Fig. 4 for the six most representative simulations. In order to remove the large scattering in the direct calculations, we perform a running mean over five frequency bins. The results of this averaging are shown by dot-dashed lines. The choice of five frequency bins is somewhat arbitrary. However we notice that between 2 to 10 frequency bins, the maximum and the shape of the spectrum do not significantly change.

Comparisons between direct and semi-analytical calculations using either $\chi_{k}^{\mathrm{G}}$ or $\chi_{k}^{\mathrm{E}}$ all show systematic differences: the excitation rates obtained with the direct calculations are systematically lower than those resulting from the semi-analytical method. These systematic differences are likely due to the too low spatial resolution of the $3 \mathrm{D}$ simulations which are used here (see Sect. 5.2 below).

At high frequency, the use of $\chi_{k}^{\mathrm{E}}$ instead of $\chi_{k}^{\mathrm{G}}$ results in larger $\mathcal{P}$ for all stars. This arises from the fact that $\chi_{k}^{\mathrm{E}}$ spreads slightly more energy at high frequency than $\chi_{k}^{\mathrm{G}}$ does (see Fig. 2).
The largest difference between the two types of calculation (direct versus semi-analytical) is seen in the case of Procyon. Indeed, the simulation of Procyon shows a pronounced depression around $v \sim 1.5 \mathrm{mHz}$. Such a depression is not seen in the semi-analytical calculations. The origin of this depression has not been clearly identified yet but is perhaps related to some interference between the turbulence and the acoustic waves which manifests itself in the pressure fluctuations in the 3D work integral but is not included in the semi-analytical description.

\subsection{Influence of the $3 D$ simulation characteristics}

In order to assess the influence of the spatial resolution of the simulation on our results, we have at our disposal three other solar 3D simulations, with a grid of $253 \times 253 \times 163,125 \times$ $125 \times 82$ and $50 \times 50 \times 82$ (hereafter S1), and a duration of $\sim 42 \mathrm{~min}, 70 \mathrm{~min}$ and $100 \mathrm{~min}$, respectively.

We have computed the $\mathrm{p}$-modes excitation rates according to the direct method for those three simulations. For each of those simulations we have also computed the excitation rates according to the semi-analytical method assuming either a Lorentzian $\chi_{k}$ or a Gaussian $\chi_{k}$.

As shown in Fig. 5 (top), the excitation rates computed according to the direct calculation increase as the spatial resolution of the 3D simulation increases. The excitation rates computed with the 3D simulations with the two highest spatial resolutions reach approximately the same mean amplitude level, indicating that this level of spatial resolution is sufficient for the direct calculations.

We note that as the spatial resolution increases, the semianalytical calculations using a Lorentzian $\chi_{k}$ decrease by a factor $\sim 2$ (not shown here). The differences in the semi-analytical calculations based on the $253 \times 253 \times 163$ simulation and the $125 \times 125 \times 82$ simulation are found very small, indicating that this level of spatial resolution is sufficient for the semi-analytical calculations too.

Finally, we note that the excitation rates obtained for the $50 \times 50 \times 82$ solar simulation (S1) are approximatively two times smaller than excitation rates for the $50 \times 50 \times 82$ solar simulation otherwise used throughout this work (S0 hereafter). This difference is attributed to the fact that the two simulations do not correspond to the same realization. Indeed, as a test, we have extended the duration of the simulation S1 up to $500 \mathrm{~min}$. The full time series has then been divided into subsets of equal duration of 100 min and p-mode excitation rates have been computed for each subset. We find that the maximum in the p-modes excitation rates $\mathcal{P}(v)$ oscillates from a subset to another about a mean value. The observed variations are large: the maximum in $\mathcal{P}(v)$ can be larger (smaller resp.) by $\sim 1.5$ ( 0.5 resp.) times the maximum in the power spectrum obtained by averaging the power spectra of all subsets. Hence we find that at low spatial resolution, different realizations yield excitation rates that are scattered about a mean value at each frequency. This dispersion is likely to be responsible for the factor of two difference between the excitation rate maxima obtained for the two realizations S0 and S1. This type of dependency of $\mathcal{P}$ - with the starting time of the time series and its duration - is expected to be smaller for simulations with resolution higher than $50 \times 50 \times 82$, because of the larger number of excitation sources there. This will be studied in a subsequent work. 

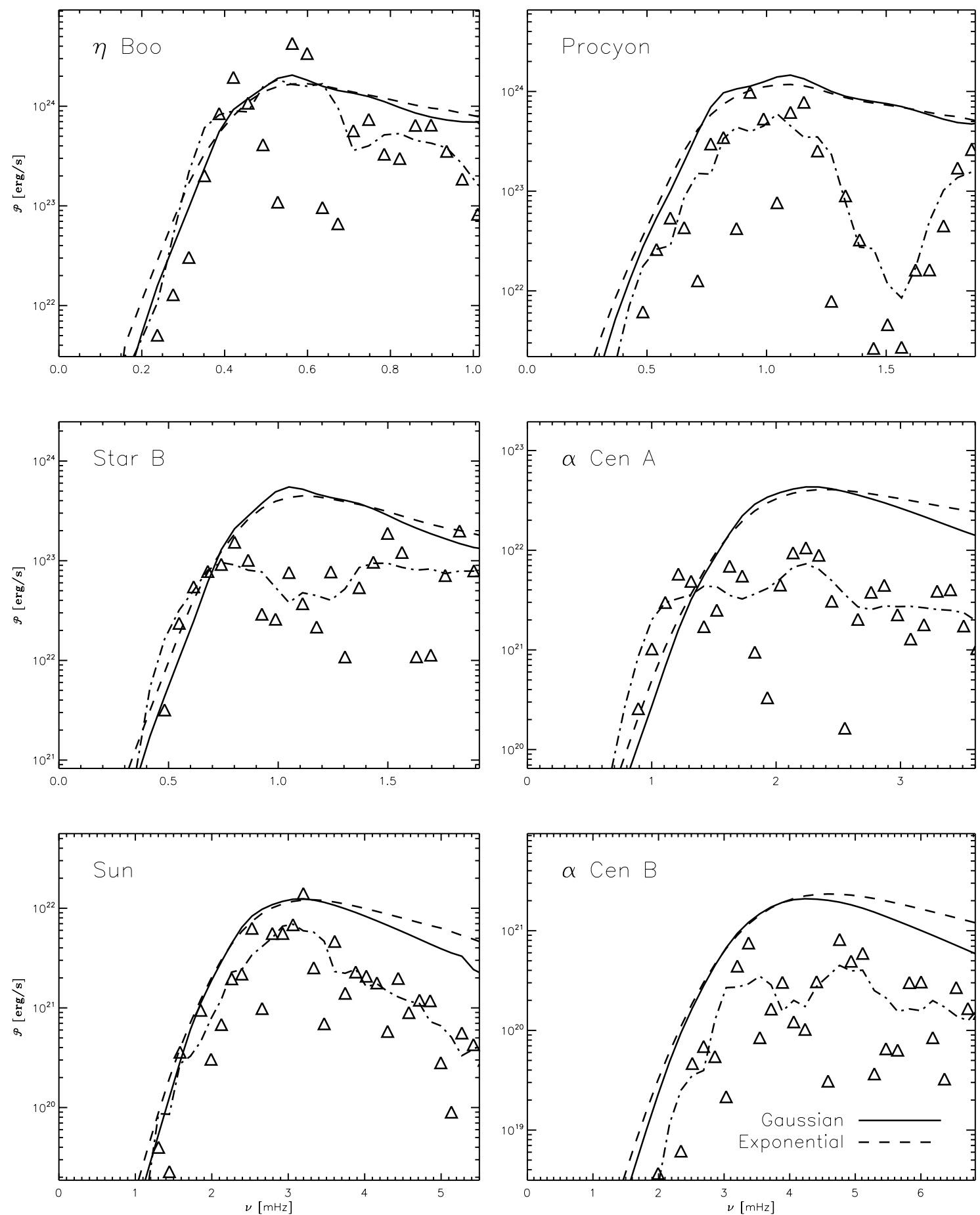

Fig. 4. Excitation rates, $\mathcal{P}$, are presented as functions of mode frequency for six of the seven convection simulations listed in Tables 1 and 2 . Each triangle corresponds to a single evaluation of the 3D work integral estimated for a given eigenfrequency according to Eq. (1). The dot-dashed lines correspond to a running mean of the triangle symbols performed over five frequencies. The solid and dashed lines correspond to the excitation rates calculated with the semi-analytical method and using the Gaussian and the exponential forms of $\chi_{k}$, respectively. All results shown are obtained as the sum of contributions from the two sources of excitation: excitation by the turbulent pressure and excitation by the non-adiabatic gas pressure.

\subsection{Eddy-time correlation: Lorentzian versus Gaussian}

As seen in Sect 5.2 above, the characteristics of the simulations influence the semi-analytical calculations of the mode excitation rates (through the input parameters which enter the semianalytical calculations and which are taken from the 3D simulation). We want to compare the results of the semi-analytical calculations using $\chi_{k}^{\mathrm{L}}$ with the semi-analytical calculations using
$\chi_{k}^{\mathrm{G}}$. It is then necessary to insert the 3D inputs in these calculations coming from simulations with the highest quality, here the highest available resolution.

Figure 5 (bottom) compares semi-analytical calculations using a Lorentzian $\chi_{k}$ with those using a Gaussian $\chi_{k}$. All theses semi-analytical calculations are here based on the energy spectrum of the simulation with the spatial resolution of $253 \times 253 \times$ 163 (see Sect. 5.2). 

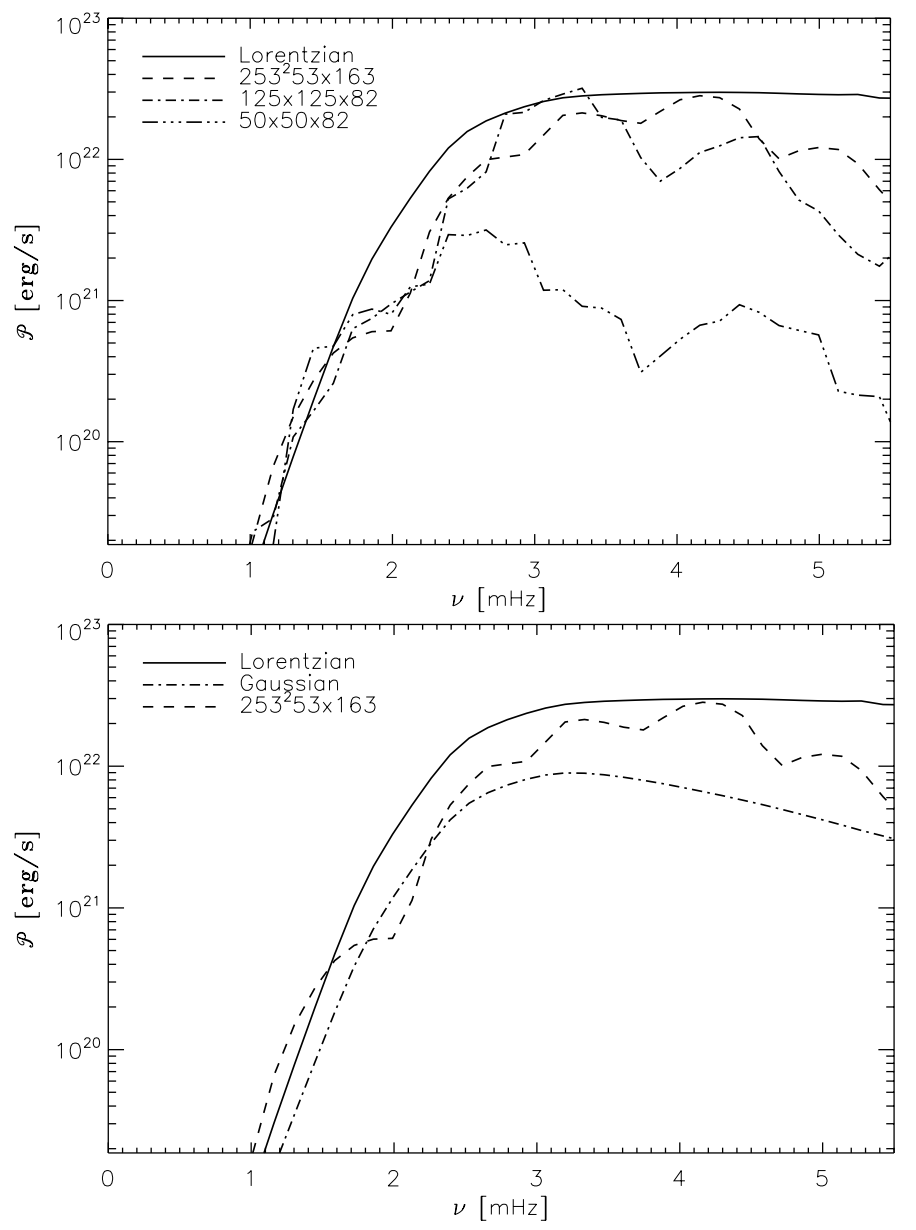

Fig. 5. Top: as in Fig. 4 for solar simulations only. The solid line corresponds to the semi-analytical calculations based on a Lorentzian $\chi_{k}$ and a simulation with a spatial resolution of $253 \times 253 \times 82$. The other lines are running means over five frequencies of the direct calculation based on solar simulations with different spatial resolution: $253 \times 253 \times 82$ (dashed line), $125 \times 125 \times 82$ (dot dashed line) and $50 \times 50 \times 82$ (dot dot dashed line). Bottom: the solid and dashed lines have the same meaning as in the top figure. The dot-dashed line corresponds to the semianalytical calculations based on a Gaussian $\chi_{k}$.

The average level of the excitation rates calculated according to the direct method and with the simulation with the highest spatial resolution is in between the semi-analytical calculations based on Lorentzian $\chi_{k}$ and those based on a Gaussian $\chi_{k}$, nevertheless they are in general slightly closer to the semi-analytical calculations based on Lorentzian $\chi_{k}$. This result is discussed in Sect. 6.2.1.

\subsection{Maximum of $\mathcal{P}$ as a function of $L / M$}

Figure 6 shows $\mathcal{P}_{\max }$, the maximum in $\mathcal{P}$, as a function of $L / M$ for the direct and the semi-analytical calculations.

The same systematic differences between the direct and the semi-analytical calculations as seen in Fig. 4 are of course observed here. Note that the differences slightly decrease with increasing values of $L / M$.

We have also computed the excitation rate with the semianalytical method using $\chi_{k}^{\mathrm{L}}$. The maximum excitation rate as evaluated with $\chi_{k}^{\mathrm{L}}$ is systematically larger than both the direct calculations and the semi-analytical results based on $\chi_{k}^{\mathrm{G}}$ or $\chi_{k}^{\mathrm{E}}$.

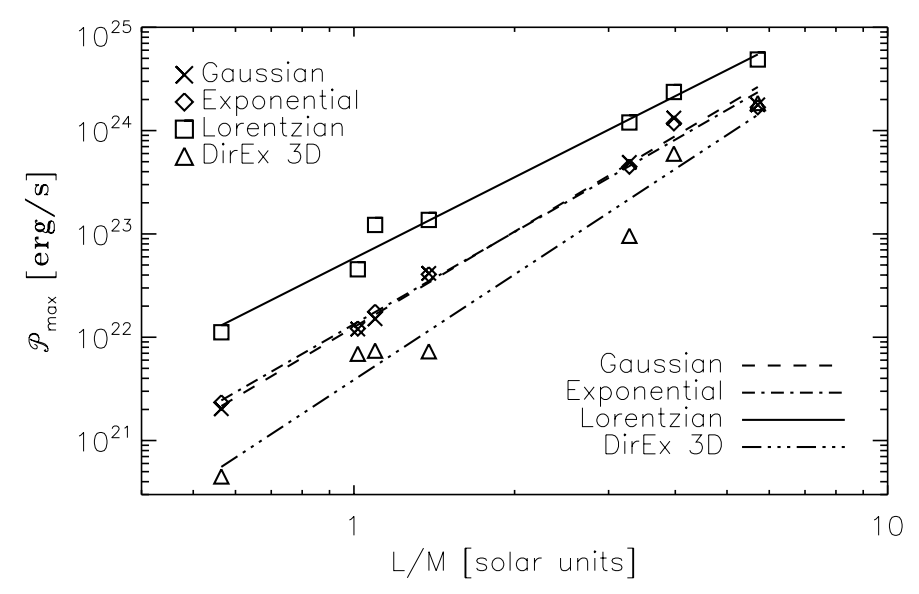

Fig. 6. $\mathcal{P}_{\max }$ versus $L / M$ where $L$ is the luminosity and $M$ is the mass of the $1 \mathrm{D}$ models associated with the 3D simulations. The triangles correspond to the direct calculations (labeled as "DirEx 3D" in the legend), and the other symbols correspond to the semi-analytical calculations using the three forms of $\chi_{k}$ : the crosses assume a Gaussian, the diamonds an exponential and the squares a Lorentzian, respectively. Each set of $\mathcal{P}_{\max }$ is fitted by a power law of the form $(L / M)^{s}$ where $s$ is the slope of the power law. The line-styles correspond to the three semi-analytical cases and the direct calculations, as indicated in the lower right corner of the plot.

Table 3. Values found for the slopes $s$ (see Sect. 5.4) and $s v$ (see Sect. 5.5). "Method" is the method considered for the calculations of $\mathcal{P}$.

\begin{tabular}{cccc}
\hline \hline Method & $\chi_{k}$ & $s$ & $s v$ \\
\hline direct & - & 3.4 & - \\
semi-analytical & Gaussian & 3.1 & 1.0 \\
semi-analytical & exponential & 3.0 & 0.9 \\
semi-analytical & Lorentzian & 2.6 & 0.7 \\
\hline
\end{tabular}

In the solar case, $\mathcal{P}_{\max }$ is found to be closer to the value derived from recent helioseismic data (Baudin et al. 2005) when using a Lorentzian compared to a Gaussian (see also Belkacem et al. 2006b, B06b hereafter). The "observed" excitation rates are derived from the velocity observations $V$ as follows:

$\mathcal{P}=2 \pi \Gamma_{v} \mathcal{M}(h) V^{2}$

where $\mathcal{M}$ is the mode mass, $V$ is the mode velocity amplitude and $h$ is the height above the photosphere where the mode mass is evaluated. The mode line width at half maximum in $\mathrm{Hz}, \Gamma_{v}=$ $\eta / \pi,\left(\eta\right.$ is the mode amplitude damping rate in $\left.\mathrm{s}^{-1}\right)$ is determined observationally in the solar case.

Using the recent helioseismic measurements of $V$ and $\Gamma_{v}$ by Baudin et al. (2005) and the mode mass computed here for our solar model at the height $h=340 \mathrm{~km}$ (cf. Baudin et al. 2005), we find $\mathcal{P}_{\text {max }, \odot}=6.5 \pm 0.7 \times 10^{22} \mathrm{erg} \mathrm{s}^{-1}$. This value must be compared with those found with $\chi_{k}^{\mathrm{L}}$ and $\chi_{k}^{\mathrm{G}}$, namely $\mathcal{P}_{\max , \odot}^{\mathrm{L}}=$ $4.9 \times 10^{22} \mathrm{erg} \mathrm{s}^{-1}$ and $\mathcal{P}_{\max , \odot}^{\mathrm{G}}=1.2 \times 10^{22} \mathrm{erg} \mathrm{s}^{-1}$ respectively.

Scaling laws: All sets of calculations can be reasonably well fitted with a scaling law of the form $\mathcal{P}_{\max } \propto(L / M)^{s}$ where $s$ is a slope which depends on the considered set of calculations. Values found for $s$ are summarized in Table 3.

- For the semi-analytical calculations, we find $s=2.6$ using $\chi_{k}^{\mathrm{L}}, s=3.0 \mathrm{using} \chi_{k}^{\mathrm{E}}$ and $s=3.1$ for the Gaussian form. 
The Lorentzian form results in a power law with a smaller slope than the Gaussian. This can be understood as follows: a Gaussian decreases more rapidly with $v$ than a Lorentzian. As the ratio $L / M$ of a main sequence star increases, the mode frequencies shift to lower values. Hence $\mathrm{p}$-modes of stars with large values of $L / M$ receive relatively more acoustic energy when adopting a Gaussian rather than a Lorentzian $\chi_{k}$. It is worthwhile to note that even though the ratio $L / M$ is the ratio of two global stellar quantities, it nevertheless characterizes essentially the stellar surface layers where the mode excitation is located since $L / M \propto T_{\text {eff }}^{4} / g$.

- For the set of direct calculations, some scatter exists as a consequence of the large statistical fluctuations in $\mathcal{P}_{\max }$ and a linear regression gives $s=3.4$. As expected, this value is rather close to that found with the semi-analytical calculations using either $\chi_{k}^{\mathrm{G}}$ or $\chi_{k}^{\mathrm{E}}$.

\subsection{Maximum of the mode amplitudes $\left(V_{\max }\right)$ as a function of $L / M$}

The theoretical oscillation velocity amplitudes $V$ can be computed according to Eq. (9) The calculation requires the knowledge of the excitation rates, $\mathcal{P}$, damping rates, $\eta$, and mode mass, $\mathcal{M}$. Although it is possible - in principle - to compute the convective dampings from the $3 \mathrm{D}$ simulations (Nordlund \& Stein 2001), it is a difficult task which is under progress. However, using for instance Gough's Mixing-Length Theory (1976; 1977, G'MLT hereafter), it is possible to compute $\eta$ and $\mathcal{P}$ for different stellar models of given $L, M$ and deduce $V_{\max }$, the maximum of the mode amplitudes, as a function of $L / M$ at the cost of some inconsistencies.

In Samadi et al. (2001), calculations of the damping rates $\eta$ based on G'MLT were performed for stellar models with different values of $L$ and $M$. Although these stellar models are not the same as those considered here, it is still possible, for a crude estimate, to determine the dependency of $V_{\max }$ with $L / M$.

Hence we proceed as follows: for each stellar model computed in Samadi et al. (2001), we derive the values of $\eta$ and $\mathcal{M}$ at the frequency $v_{\max }$ at which the maximum amplitude is expected. From the stars for which solar-like oscillations have been detected, Bedding \& Kjeldsen (2003) have shown that this frequency is proportional to the cut-off frequency. Hence we determine $v_{\max }=\left(v_{\mathrm{c}} / v_{\mathrm{c}, \odot}\right) v_{\max , \odot}$ where $v_{\mathrm{c}}$ is the cut-off frequency of a given model and the symbol $\odot$ refers to solar quantities $\left(v_{\text {max }, \odot} \simeq 3.2 \mathrm{mHz}\right.$ and $\left.v_{c, \odot} \simeq 5.5 \mathrm{mHz}\right)$. We then obtain $\left(\eta_{\max } \mathcal{M}_{\max }\right)$ as a function of $L$ and $M$.

On the other hand, in Sect. 5.4, we have established $\mathcal{P}_{\max }$ as a function of $L$ and $M$. Then, according to Eq. (9), we can determine $V_{\max }(L, M)$ for the different power laws of $\mathcal{P}_{\max }$.

We are interested here in the slope (i.e. variation with $L / M$ ) of $V_{\max }$ and not its absolute magnitude, therefore we scale the theoretical and observed $V_{\max }$ with a same normalization value which is taken as the solar value $V_{\max , \odot}=33.1 \pm 0.9 \mathrm{~cm} \mathrm{~s}^{-1}$ as determined recently by Baudin et al. (2005).

We find that $V_{\max }$ increases as $(L / M)^{s v}$ with different values for $s v$ depending on the assumptions for $\chi_{k}$. The values of $s v$ are summarized in Table 3 and illustrated in Fig. 7. We find $s v \simeq 0.7$ with $\chi_{k}^{\mathrm{L}}$ and $s v \simeq 1.0$ with $\chi_{k}^{\mathrm{G}}$.

These scaling laws must be compared with observations of a few stars for which solar-like oscillations have been detected in Doppler velocity. The observed $V_{\max }$ are taken from Table 1 of HG02, except for $\eta$ Boo, $\zeta$ Her A, $\beta$ Vir, HD 49933 and $\mu$ Ara, for which we use the $V_{\max }$ quoted by Carrier et al. (2003), Martić et al. (2001), Martić et al. (2004), Mosser et al. (2005) and

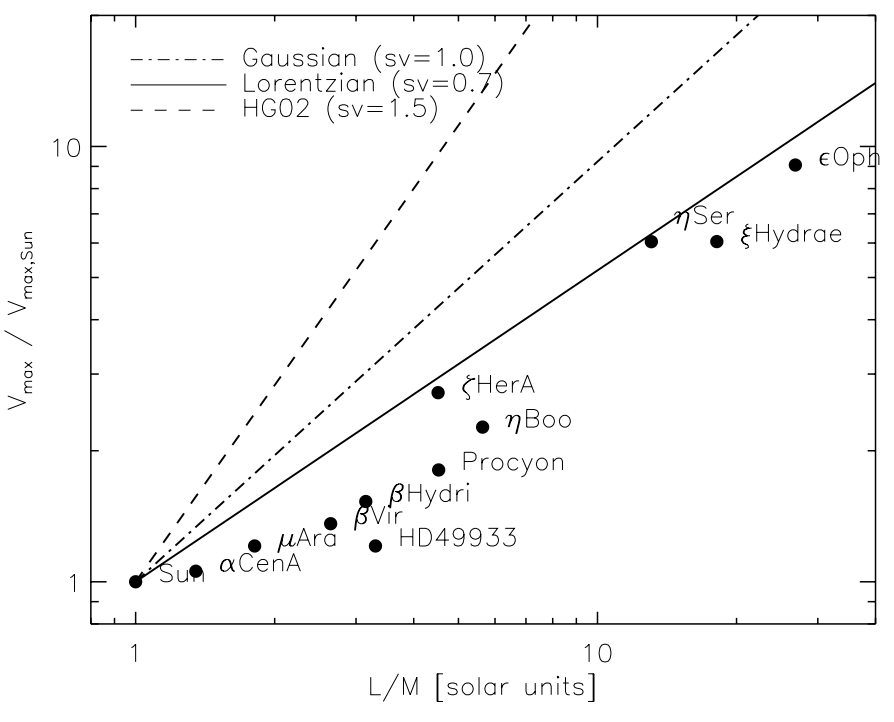

Fig. 7. Same as Fig. 6 for $V_{\max } / V_{\max , \odot}$, the maximum of the mode amplitudes relative to the observed solar value $\left(V_{\max , \odot}=33.1 \pm 0.9 \mathrm{~cm} \mathrm{~s}^{-1}\right)$. The filled symbols correspond to the stars for which solar-like oscillations have been detected in Doppler velocity. The lines - except the dashed line - correspond to the power laws obtained from the predicted scaling laws for $\mathcal{P}_{\max }$ (Fig. 6) and estimated values of the damping rates $\eta_{\max }$ (see text for details). Results for two different eddy timecorrelation functions, $\chi_{k}$, are presented: Lorentzian (solid line) and Gaussian (dot-dashed line) functions. For comparison the dashed line shows the result by HG02. Values of the slope $s v$ are given on the plot and in Table 3.

Bouchy et al. (2005) respectively and $\epsilon$ Oph and $\eta$ Ser quoted by Barban et al. (2004).

Figure 7 shows that the observations also indicate a monotonic logarithmic increase of $V_{\max }$ with $L / M$ despite a large dispersion which may at least partly arise from different origins of the data sets. For the observations we find a "slope" $s v \simeq 0.7$. This is close to the theoretical slope obtained when adopting $\chi_{k}^{\mathrm{L}}$ and definitely lower than the slopes obtained when adopting $\chi_{k}^{\mathrm{G}}$ or adopted by HG02.

\section{Summary and discussion}

One goal of the present work has been to validate the model of stochastic excitation presented in Paper I. The result of this test is summarized in Sect. 6.1. A second goal has been to study the properties of the turbulent eddy time-correlation, $\chi_{k}$, and the importance for the calculation of the excitation rates, $\mathcal{P}$, of the adopted form of $\chi_{k}$. Section 6.2 deals with this subject.

\subsection{Validation of the excitation model}

In order to check the validity of the excitation model, seven 3D simulations of stars, including the Sun, have been considered. For each simulation, we calculated the p-mode excitation rates, $\mathcal{P}$, using two methods: the semi-analytical excitation model (cf. Sect. 2.2) that we are testing, and a direct calculation as detailed in Sect. 2. In the latter method, the work performed by the pressure fluctuations on the p-modes is calculated directly from the 3D simulations.

In the semi-analytical method, $\mathcal{P}$ is computed according to the excitation model of Paper I. The calculation uses, as input, information from the 3D simulations as for instance the eddy 
time-correlation $\left(\chi_{k}\right)$ and the kinetic energy spectra $(E(k))$. However although $\chi_{k}$ has been computed for each simulation, in practice for simplifying the problem of implementation as well as for comparison purpose with Paper III, we chose to represent the $v$ variation of $\chi_{k}$ with simple analytical functions. It is found that the $v$-variation of $\chi_{k}$ in the present simulations lies loosely between that of an exponential and a Gaussian. We then perform the validation test of the excitation model using those two forms of $\chi_{k}$.

We find that using either $\chi_{k}^{\mathrm{G}}$ or $\chi_{k}^{\mathrm{E}}$ in the semi-analytical calculations of $\mathcal{P}$ results in systematically higher excitation rates than those obtained with direct 3D calculations. These systematic differences are attributed to the low spatial resolution of our present set of simulations. Indeed we have shown here that using solar simulations with different spatial resolutions, the resulting excitation rates increase with increasing spatial resolution.

We have next investigated the dependence of $\mathcal{P}_{\max }$ with $L / M$ (See Fig. 6), where $L$ and $M$ are the stellar luminosity and mass respectively. As in previous works based on a purely theoretical approach (e.g. Samadi et al. 2003a), we find that $\mathcal{P}_{\max }$ scales approximatively as $(L / M)^{s}$ where $s$ is the slope of the scaling law: we find $s=3.4$ with the direct calculations and $s=3.2$ and $s=3.1$ with the semi-analytical calculations using $\chi_{k}^{\mathrm{G}}$ and $\chi_{k}^{\mathrm{E}}$ respectively. This indicates a general agreement between the scaling properties of both types of calculations, which validates to some extent the adopted excitation model across the domain of the HR diagram studied here.

For the sake of simplicity, only simple analytical forms for $\chi_{k}$ have been investigated here. We expect that the use of more sophisticated forms for $\chi_{k}$ would reduce the dispersion between the analytical and direct calculations, but would not affect the conclusions of the present paper.

\subsection{The eddy time-correlation spectra, $\chi_{\mathrm{k}}$}

The slope $s$ of the scaling law for $\mathcal{P}_{\max }$, is found to depend significantly on the adopted analytical form for $\chi_{k}$. The semianalytical calculations using the Lorentzian form for $\chi_{k}$ results in a significantly smaller slope $s$ than those based on the Gaussian or the exponential or from direct calculations (see Table 3).

Except for the Sun, independent and accurate enough constraints on both the mode damping rates and the mode excitation rates are not yet available. We are then left to perform comparison between predicted and observed mode amplitudes. Unfortunately, obtaining tight constraints on $\chi_{k}$ using comparison between predicted and observed mode amplitudes is hampered by large uncertainties in the theoretical estimates of the damping rates. It is therefore currently difficult to derive the excitation rates $\mathcal{P}$ for the few stars for which solar-like oscillations have been detected (see Samadi et al. 2004). The future space mission COROT (Baglin \& The Corot Team 1998) will provide high-quality data on seismic observations. Indeed the COROT mission will be the first mission that will provide both high precision mode amplitudes and line-widths for stars other than the Sun. It will then be possible to use the observed damping rates and to derive the excitation rate $\mathcal{P}$ free of the uncertainties associated with a theoretical computation of damping rates. In particular, it will be possible to determine $\mathcal{P}_{\max }$ as a function of $L$ and $M$ from the observed stars. Such observations will provide valuable constraints for our models for $\chi_{k}$.

We can, nevertheless, already give some arguments below in favor of the Lorentzian being the correct description for $\chi_{k}$.

\subsubsection{Solar case}

In the 3D simulations studied here, including that of the Sun, the inferred $v$ dependency of $\chi_{k}$ is far from a Lorentzian, in contrast to that found with the solar $3 \mathrm{D}$ simulation investigated in Paper III. However, by investigating solar simulations with different resolutions, we find that, as the spatial resolution increases, $\chi_{k}$ tends towards a Lorentzian $v$-dependency. This explanation is likely to stand for non-solar simulations too, but has not yet been confirmed (work in progress).

Furthermore, as shown in Fig. 5, bottom, the direct calculations obtained with the simulation with the highest spatial resolution available is slightly closer to the semi-analytical calculations using the Lorentzian form than those using the Gaussian one.

Independently of the resolution (if large enough of course), a Lorentzian $\chi_{k}$ predicts larger values for $\mathcal{P}_{\max }$ than a Gaussian or an exponential do. In particular in the solar case, the semianalytical calculation using $\chi_{k}^{\mathrm{L}}$ results in a $\mathcal{P}_{\max }$ closer to the helioseismic constraints derived by Baudin et al. (2005) compared to using $\chi_{k}^{\mathrm{G}}$ or $\chi_{k}^{\mathrm{E}}$. This latter result is in agreement with that of Paper III.

Part of the remaining discrepancies with the helioseismic constraints are attributed to the adopted closure model according to Belkacem et al. (2006b, B06b hereafter). Indeed, theoretical models of stochastic excitation adopt the quasi-normal approximation (QNA). As shown in B06b, the skew introduced by the QNA result in a under-estimation of the solar $\mathrm{p}$ mode excitation rates. When the so-called closure model with plumes proposed by Belkacem et al. (2006a) is adopted, new semi-theoretical calculations fit rather well the recent helioseismic constraints derived by Baudin et al. (2005, see B06b).

\subsection{2. $V_{\max }$ as a function of $L / M$}

Consequences of the predicted power laws for $\mathcal{P}_{\max }$ have also been crudely investigated here for the expected value of $V_{\max }$, the maximum value of the mode velocity (Fig. 7). Calculations of $V_{\max }$ from $\mathcal{P}_{\max }$ require the knowledge of the mode damping rates, $\eta$, which cannot be fully determined from the simulations. We are then led to use theoretical calculations of the damping rates. We consider here those performed by Samadi et al. (2001) which are based on Gough's $(1976 ; 1977)$ non-local and timedependent formulation of convection. From those values of $\eta$ and the different power laws for $\mathcal{P}_{\max }$ expected values of $V_{\max }$ are obtained.

We find, as in Houdek \& Gough (2002, HG02), that $V_{\max }$ scales as $(L / M)^{s v}$. Calculations by HG02 result in $s v \simeq 1.5$. Our semi-analytical calculations of $\mathcal{P}_{\max }$ based on a Gaussian $\chi_{k}$ result in a slightly smaller slope $(s v \simeq 1.0)$. On the other hand, using a Lorentzian $\chi_{k}$ results in a slope $s v \simeq 0.7$ which is closer to that derived from the few stars for which oscillation amplitudes have been measured.

From this result, we conclude that the problem of the overestimation of the amplitudes of the solar-like oscillating stars more luminous than the Sun is related to the choice of the model for $\chi_{k}$. Indeed, previous theoretical calculations by Houdek et al. (1999) are based on the assumption of a Gaussian $\chi_{k}$. As shown here, the Gaussian assumption results in a larger slope $s v$ than the Lorentzian $\chi_{k}$. This is the reason why Houdek et al. (1999) over-estimate $V_{\max }$ for $L / M>L_{\odot} / M_{\odot}$.

On the other hand, if one assumes $\chi_{k}=\chi_{k}^{\mathrm{L}}$, a scaling factor is no longer required to reproduce $\mathcal{P}_{\max }$ for the solar $\mathrm{p}$-modes. Moreover, as a consequence of the smaller slope, $s v$, resulting 
from a Lorentzian $\chi_{k}$, the predicted amplitudes for other stars match the observations better.

This result further indicates that a Lorentzian is the better choice for $\chi_{k}$, as was also concluded in Paper III.

Departures of the theoretical curve from the observed points in Fig. 7 can be attributed to several causes which remain to be investigated:

1) A major uncertainty comes from the computed damping rates as no accurate enough observations are available yet to validate them. As $V$ results from the balance between $\mathcal{P}$ and $\eta$, the slope $s v$ can also depend on the variation of $\eta$ with $L / M$. Thus, the large differences in $s v$ between the seismic observations and the calculations based on $\chi_{k}^{\mathrm{G}}$ can also be, a priori, attributed to an incorrect evaluation of the damping rates. However $\eta_{\max }-$ the value of the damping rate at the frequency $v_{\max }$ at which the maximum amplitude is expected - does not follow a clear scaling law with $L / M$. We have looked at the $\eta_{\max }$ variation in our set of G'MLT models and found no clear dependence of $\eta_{\max }$ on $L / M$ but rather a dispersion.

2) The observed stars in Fig. 7 have somewhat different chemical compositions; this can cause some scatter in the relation $V_{\max }-L / M$ which has not been taken into account here. All the simulations investigated in the present work employ a solar metal abundance. The metallicity has a direct impact on the opacity and the EOS. Both in turn affect the internal structure and are also decisive for the transition from convection to radiation in the photosphere and therefore determine the structure of the super-adiabatic region. Hence, the properties of the super-adiabatic region, relevant for the excitation rates, differ for stars located at the same position in the HR diagram (e.g., same $T_{\text {eff }}$ and same $g$ ) but with different metal abundances. Consequently the excitation of p-modes for such stars probably differ, although it remains to be seen to what extent. A differential investigation of the metallicity effect is planned for the future.

\subsection{Relative contribution of the turbulent pressure}

Another issue concerns the relative contribution of the turbulent pressure. The excitation of solar-like oscillations is generally attributed to the turbulent pressure (i.e. Reynolds stress) and the entropy fluctuations (i.e. non-adiabatic gas pressure fluctuations) and occurs in the super-adiabatic region where those two terms are the largest. In Paper III, it was found that the two driving sources are of the same order of magnitude, in contradiction with the results by Stein \& Nordlund (2001) who found - based on their 3D numerical simulations of convection - that the turbulent pressure is the dominant contribution to the excitation. The discrepancy is removed here as we used a corrected version of the formulation of the contribution of the Reynolds stress of Paper I (see Eq. (3)), leading to a larger contribution from the Reynolds stress.

For the Sun, assuming $\chi_{k}^{\mathrm{L}}\left(\chi_{k}^{\mathrm{G}}\right.$ resp.), we now find that the Reynolds stress contribution is 5 times ( 3 times resp.) larger than that due to the entropy fluctuations (non-adiabatic gas-pressure fluctuations). Hence, the Reynolds stress is indeed the dominant source of excitation in agreement with the results of Stein \& Nordlund (2001). The best agreement with the latter results is obtained with a Lorentzian $\chi_{k}$.

However, we find that the relative contribution from Reynolds stresses decreases rapidly with $(L / M)$. For instance, in the simulation of Procyon, the Reynolds stress represents only $\sim 30 \%$ of the total excitation rate.

From that, we conclude that the excitation by entropy fluctuations cannot be neglected, especially for stars more luminous than the Sun.

Acknowledgements. R.S.'s work has been supported by Société de Secours des Amis des Sciences (Paris, France) and by Fundacão para a Ciência e a Tecnologia (Portugal) under grant SFRH/BPD/11663/2002. R.F.S. is supported by NASA grants NAG 512450 and NNG04GB92G and by NSF grant AST0205500. We thank the referee, Mathias Steffen, for his judicious suggestions which helped improve this manuscript.

\section{Appendix A: Calculation of the non adiabatic pressure fluctuations}

The adiabatic variation of the gas pressure does not contribute to the $\Delta(P \mathrm{~d} V)$ work over an oscillation period as it is in phase with the volume (or density) variation. In practice, however, it is beneficial for the accuracy of the computation of excitation to subtract the adiabatic part of the gas pressure fluctuation, since it reduces the coherent part. That part gives zero contribution only in the limit of infinite time, or for an exact integer number of periods. However, in practice, it gives rise to a random (or noisy) contribution. Indeed, as we deal with a lot of different modes it is hard to find a time-interval which is an integer number of periods of each and all of the modes at the same time.

The Lagrangian variations of gas pressure, $\Delta P_{\text {gas }}$ must satisfy

$\Delta P_{\mathrm{gas}}=\frac{\Gamma_{1} P_{\mathrm{gas}}}{\rho} \Delta \rho+\frac{\partial P_{\mathrm{gas}}}{\partial S} \Delta S$

where $P_{\text {gas }}, \rho$ and $S$ are the gas pressure the density and the entropy respectively and where the operator $\Delta$ represents the pseudo Lagrangian fluctuations of a given quantity. The concept of pseudo Lagrangian fluctuations is introduced in Nordlund \& Stein (2001). Accordingly we derive the non-adiabatic gas pressure fluctuations as:

$\Delta P_{\text {gas,nad }}(\boldsymbol{r}, t) \equiv \Delta P_{\text {gas }}-c_{\mathrm{s}}^{2} \Delta \rho$

where $c_{\mathrm{s}}^{2} \equiv \Gamma_{1} P_{\text {gas }} / \rho$ is the sound speed.

However, what we want to subtract off from $\Delta P_{\text {gas }}$ is that part of the pressure variation that is due to adiabatic compression and expansion due to the particular radial wave modes (i.e. the low amplitude perturbation of $\rho(r)$ on top of the possibly large variations horizontally of $\rho(\boldsymbol{r})$ that $\rho(r)$ is an average of).

To find the nonadiabatic pressure fluctuations, we start with calculations of horizontal averages of the primary quantities, $P_{\text {gas }}, P_{\text {turb }}, \rho$ and $c_{\mathrm{s}}^{2}$. We convert these averages to the pseudoLagrangian frame of reference, in which the net mass flux vanishes. We then compute fluctuations of the resulting quantities with respect to time, i.e., subtract their time averages:

$$
\begin{array}{r}
\Delta P_{\text {gas }}=\left\langle P_{\text {gas }}\right\rangle_{\mathrm{h}}-\left\langle P_{\text {gas }}\right\rangle_{\mathrm{h}, \mathrm{t}} \\
\Delta P_{\text {turb }}=\left\langle P_{\text {turb }}\right\rangle_{\mathrm{h}}-\left\langle P_{\text {turb }}\right\rangle_{\mathrm{h}, \mathrm{t}} \\
\Delta \rho=\langle\rho\rangle_{\mathrm{h}}-\langle\rho\rangle_{\mathrm{h}, \mathrm{t}} .
\end{array}
$$

Here, \langle\rangle$_{\mathrm{h}}$ refers to horizontal average and \langle\rangle$_{\mathrm{h}, \mathrm{t}}$ refers to consequent time average performed on a horizontally averaged quantity. Finally, the non-adiabatic fluctuations of the total pressure (that is gas + turbulent pressure) are:

$$
\begin{aligned}
\Delta P_{\mathrm{nad}}= & \Delta P_{\mathrm{gas}, \mathrm{nad}}+\Delta P_{\mathrm{turb}} \\
& =\Delta P-\left\langle c_{s}^{2}\right\rangle_{\mathrm{h}, \mathrm{t}} \Delta \rho
\end{aligned}
$$

where $\Delta P \equiv \Delta P_{\text {gas }}+\Delta P_{\text {turb }}$. 


\section{References}

Baglin, A., \& The Corot Team. 1998, in IAU Symp.: New Eyes to See Inside the Sun and Stars, 185, 301

Balmforth, N. J. 1992, MNRAS, 255, 639

Barban, C., de Ridder, J., Mazumdar, A., et al. 2004, in Proceedings of the SOHO 14 / GONG 2004 Workshop (ESA SP-559): Helio- and Asteroseismology: Towards a Golden Future, ed. D. Danesy, 113

Baudin, F., Samadi, R., Goupil, M.-J., et al. 2005, A\&A, 433, 349

Bedding, T. R., \& Kjeldsen, H. 2003, Pub. Astron. Soc. Aust., 20, 203

Belkacem, K., Samadi, R., Goupil, M., \& Kupka, F. 2006a, A\&A, 460, 173

Belkacem, K., Samadi, R., Goupil, M., \& Kupka, F. 2006b, A\&A, 460, 183 (B06b)

Böhm-Vitense, E. 1958, Z. Astrophys., 46, 108

Bouchy, F., Bazot, M., Santos, N. C., Vauclair, S., \& Sosnowska, D. 2005, A\&A, 440, 609

Carrier, F., Bouchy, F., \& Eggenberger, P. 2003, in Asteroseismology across the HR diagram, ed. M. J. Thompson, M. S. Cunha, \& M. J. P. F. G. Monteiro, CDROM (Kluwer Acad.), 284315

Christensen-Dalsgaard, J. 1982, MNRAS, 199, 735

Christensen-Dalsgaard, J., \& Berthomieu, G. 1991, Theory of solar oscillations, Solar interior and atmosphere (A92-36201 14-92) (Tucson, AZ: University of Arizona Press), 401

Christensen-Dalsgaard, J., \& Frandsen, S. 1983a, Sol. Phys., 82, 165

Christensen-Dalsgaard, J., \& Frandsen, S. 1983b, Sol. Phys., 82, 469

Däppen, W., Mihalas, D., Hummer, D. G., \& Mihalas, B. W. 1988, ApJ, 332, 261

Georgobiani, D., Stein, R., \& Nordlund, A. 2006, ApJ (submitted)

Goldreich, P., \& Keeley, D. A. 1977, ApJ, 212, 243

Goldreich, P., Murray, N., \& Kumar, P. 1994, ApJ, 424, 466

Gough, D. 1976, in Lecture notes in physics, Problems of stellar convection, ed. E. Spiegel, \& J.-P. Zahn (Springer Verlag), 71, 15

Gough, D. O. 1977, ApJ, 214, 196

Houdek, G., Balmforth, N. J., Christensen-Dalsgaard, J., \& Gough, D. O. 1999, A\&A, 351, 582

Houdek, G., \& Gough, D. O. 2002, MNRAS, 336, L65 (HG02)

Kjeldsen, H., \& Bedding, T. R. 1995, A\&A, 293, 87

Kjeldsen, H., \& Bedding, T. R. 2001, Proceedings of the SOHO 10/GONG 2000 Workshop (ESA SP): Helio- and asteroseismology at the dawn of the millennium, ed. A. Wilson, 464, 361
Kurucz, R. L. 1992a, Rev. Mex. Astron. Astrofis., 23, 45

Kurucz, R. L. 1992b, Rev. Mex. Astron. Astrofis., 23, 181

Martić, M., Lebrun, J. C., Schmitt, J., Appourchaux, T., \& Bertaux, J. L. 2001, in ESA SP SOHO 10/GONG 2000 Workshop: Helio- and Asteroseismology at the Dawn of the Millennium, 464, 431

Martić, M., Lebrun, J., Appourchaux, T., \& Schmitt, J. 2004, in Proc. of the SOHO 14 / GONG 2004 Workshop (ESA SP). Helio- and Asteroseismology: Towards a Golden Future, ed. D. Danesy, 559, 563

Mosser, B., Bouchy, F., Catala, C., et al. 2005, A\&A, 431, L13

Nordlund, Å., \& Stein, R. F. 2001, ApJ, 546, 576

Osaki, Y. 1990, in Lecture Notes in Physics: Progress of Seismology of the Sun and Stars, ed. Y. Osaki, \& H. Shibahashi (Springer-Verlag), 75

Samadi, R., \& Goupil, M. . 2001, A\&A, 370, 136 (Paper I)

Samadi, R., Houdek, G., Goupil, M.-J., Lebreton, Y., \& Baglin, A. 2001, in 1st Eddington Workshop (ESA SP-485): Stellar Structure and Habitable Planet Finding, [arXiv: astro-ph/0109174], 87

Samadi, R., Goupil, M. J., Lebreton, Y., Nordlund, Å., \& Baudin, F. 2003a, Ap\&SS, 284, 221

Samadi, R., Nordlund, Å., Stein, R. F., Goupil, M. J., \& Roxburgh, I. 2003b, A\&A, 404, 1129 (Paper III)

Samadi, R., Nordlund, Å., Stein, R. F., Goupil, M. J., \& Roxburgh, I. 2003c, A\&A, 403, 303 (Paper II)

Samadi, R., Goupil, M. J., Baudin, F., et al. 2004, in Proc. of the SOHO 14 / GONG 2004 Workshop (ESA SP): Helio- and Asteroseismology: Towards a Golden Future, ed. D. Danesy, 559, 615 [arXiv: astro-ph/0409325]

Samadi, R., Goupil, M.-J., Alecian, E., et al. 2005, JA\&A, 26, 171

Stein, R., Georgobiani, D., Trampedach, R., Ludwig, H.-G., \& Nordlund, Å. 2004, Sol. Phys., 220, 229

Stein, R. F. 1967, Sol. Phys. 2, 385

Stein, R. F., \& Nordlund, A. 2001, ApJ, 546, 585

Trampedach, R., Stein, R. F., Christensen-Dalsgaard, J., \& Nordlund, Å. 1999, in ASPC Ser., 233

Trampedach, R., Christensen-Dalsgaard, J., Nordlund, Å., \& Stein, R. F. 2006a, ApJ, submitted

Trampedach, R., Stein, R. F., Christensen-Dalsgaard, J., \& Nordlund, Å. 2006b, $\mathrm{ApJ}$, in preparation 